\title{
Perceiving is believing: a Bayesian approach to explaining the positive symptoms of schizophrenia
}

\section{Paul C. Fletcher* and Chris D. Frith ${ }^{\ddagger \S}$}

Abstract |Advances in cognitive neuroscience offer us new ways to understand the symptoms of mental illness by uniting basic neurochemical and neurophysiological observations with the conscious experiences that characterize these symptoms. Cognitive theories about the positive symptoms of schizophrenia - hallucinations and delusions have tended to treat perception and belief formation as distinct processes. However, recent advances in computational neuroscience have led us to consider the unusual perceptual experiences of patients and their sometimes bizarre beliefs as part of the same core abnormality - a disturbance in error-dependent updating of inferences and beliefs about the world. We suggest that it is possible to understand these symptoms in terms of a disturbed hierarchical Bayesian framework, without recourse to separate considerations of experience and belief.

Cognitive behavioural therapy

A form of psychotherapy in which the patient is encouraged to examine the cognitive processes by which they arrive at a particular state of mind, and to change these processes together with the accompanying behaviours that may reinforce them.

*University of Cambridge, Department of Psychiatry, Addenbrooke's Hospital, Hills Road, Cambridge, CB2 200, UK.

${ }^{\ddagger}$ Centre for Functionally Integrative Neuroscience, Aarhus University Hospital, 8000 Aarhus C, Denmark. ${ }^{\S}$ Wellcome Trust Centre for Neuroimaging, Functional Imaging Laboratory, University College London, London, WC1N 3BG, UK. Correspondence to C.D.F. e-mail:c.frith@ucl.ac.uk doi:10.1038/nrn2536 Published online

3 December 2008
Schizophrenia is not a rare disorder. It has a lifetime risk of $\sim 0.7 \%^{1}$ (similar to that of rheumatoid arthritis). It has a genetic basis, but the importance of social factors in its emergence is also recognized. Schizophrenia is devastating for both sufferers and their carers. Patients are likely to be unemployed or fail to fulfil their original potential. Contact with the police resulting from socially unacceptable behaviour is common, and the risk of suicide is high. The first episode typically occurs when patients are in their mid 20s, and most sufferers never fully recover. Although drug treatment and, more recently, cognitive behavioural therapy can reduce suffering, there is as yet no cure for this disorder. Furthermore, although schizophrenia clearly has a strong biological component (BOX 1), no diagnostic physiological markers have been found. Diagnosis, therefore, is made on the basis of symptoms described by the patient, signs observed by the clinician and the history of the disorder (BOX 2).

The most striking and characteristic features of the disorder are hallucinations and delusions. Hallucinations are false perceptions, such as patients hearing people talking about them or hearing their thoughts spoken aloud (TABLE 1). Delusions are persistent bizarre or irrational beliefs that are not easily understood in terms of an individual's social or cultural background. For example, patients may believe that other people can hear their thoughts or that the government is monitoring their every action. Hallucinations and delusions are examples of positive symptoms, which are so called because the abnormality lies in their presence. Positive symptoms contrast with negative symptoms (also known as signs), which are defined by the absence of normal functions, as is the case with reduced speech output (alogia) or loss of motivation (avolition). There is evidence that positive and negative symptoms reflect different underlying physiological disorders ${ }^{2,3}$. Although an important challenge for future work will be to find an explanation for both positive and negative symptoms, we believe that the current state of the field and the fact that these symptoms seem to dissociate across groups of patients make it sensible to confine our ideas in this Review to the positive symptoms. Our aim is to consider how abnormal physiological responses in the brains of people with schizophrenia might be linked to the positive symptoms that they experience. We show that a common mechanism, involving minimization of prediction error, may underlie perception and inference, and that a disruption in this mechanism may cause both abnormal perceptions (hallucinations) and abnormal beliefs (delusions). We are not concerned with the ultimate causes of the disorder, in which both genetic and environmental factors play a part. 


\section{Explaining positive symptoms}

Hallucinations and delusions are the features of schizophrenia that are the most difficult to understand $d^{4}$. These symptoms often place strain on a patient's relationships because the patient accepts them as real whereas others see them as extraordinary and bizarre. Several factors underlie this lack of understanding. Although many healthy people experience disorganization or a lack of motivation, they do not often experience persistent false perceptions or false beliefs similar to those described by patients (however, see BOX 3). Such experiences imply the possibility of a loss of contact with reality, which is frightening. Furthermore, although it is relatively easy to understand how a brain disorder might cause a loss of sensation or an inability to think, it is more difficult to understand how a brain disorder can create new and compelling experiences. Such explanations require one to forge a link between brain activity and the subjective experience of a mind. Explanations such as 'hallucinations are caused by overactive dopamine receptors' are unsatisfactory because they leave an explanatory gap between the mental and the physical. How can dopamine cause a voice or a belief?

A successful explanation of positive symptoms needs to work at three levels. It must identify the aberrant physical processes occurring at the neural level. It must link these with aberrant cognitive processes occurring at the psychological level. Finally, it must take into account the experiential level: that is, it must provide some insight into what it is like to experience positive symptoms. The cognitive level of explanation has a key role in this endeavour ${ }^{5}$. The language of cognitive neuroscience provides a bridge between the mental and the physical, because it uses terms derived from information processing and computational modelling that can be applied to both domains. Furthermore, the cognitive level of description constrains the development of physiological theories to ensure that they remain in contact with the psychotic experiences that they are ultimately designed to explain. We therefore begin this Review with a brief account of cognitive theories of the origins of positive symptoms.

\section{Box 1 | Evidence of a biological basis for schizophrenia}

Some biological factors are generally agreed to be relevant to schizophrenia. However, these biological factors also interact with environmental factors, such as prenatal exposure to infection ${ }^{97}$ and social disadvantage ${ }^{98}$.

Genetics

Liability to schizophrenia is highly heritable ( 0.81), and concordance between identical twins is almost $50 \%$. Several genes that increase the risk of developing the disorder have been identified, such as catechol-O-methyltransferase (COMT) and neuregulin $1(N R G 1)^{99}$.

\section{Neuropathology}

Imaging studies reveal enlarged ventricles and reduced cortical volume, especially in the medial temporal lobe ${ }^{100}$. In post-mortem studies, pyramidal neurons in input layers of the prefrontal cortex have a reduced dendritic spine density ${ }^{101}$.

Pharmacology

All drugs with established anti-psychotic effects block dopamine D2-like receptors ${ }^{102}$. Exposure to amphetamine, a dopamine agonist, can result in schizophrenia-like symptoms ${ }^{103}$, as can a single exposure to phencyclidine (PCP) or other NMDA ( $N$-methyl-D-aspartate) receptor antagonists, such as ketamine ${ }^{104,105}$.

\section{Cognitive theories of positive symptoms}

Abnormal perception or abnormal belief? Initially it seems plausible that delusions should arise from disordered reasoning - the patient failing to draw the correct conclusions from the available information. However, the evidence that deluded patients have difficulty with standard logical-reasoning tasks is limited ${ }^{6}$. Furthermore, the symptoms that are typically reported suggest something more subtle than a general problem with reasoning. Although hallucinations and delusions can be found in many neurological and psychiatric disorders, there is a subset of these symptoms (see TABLE 1 for some examples) that is more specifically related to a diagnosis of schizophrenia ${ }^{7}$. This subset of symptoms includes hallucinations and delusions that are particularly related to a sense of being passive - that is, of being subject to the control of an external force or agent.

There are obviously close connections between hallucinations and delusions, which suggests that the terms may be different labels for the same experiences. For example, a blurring of the distinction between false perceptions and false beliefs is seen in the case of delusions of control, when someone believes that their actions are caused by outside influences. Here, many (including at least one set of current diagnostic guidelines ${ }^{8}$ ) would argue that these are strange perceptions rather than strange beliefs, because the symptom is defined as the patient 'experiencing' their actions as being caused by outside influences. A major class of theories of positive symptoms - theories of abnormal perception - makes this critical link between hallucinations and delusions? According to these models, the primary cause of positive symptoms is an abnormal sensory experience (a hallucination). Delusions follow as a secondary consequence of attempts to understand the anomalous sensory experience. For example, if patients can hear their own thoughts being spoken aloud (hallucination), it would seem logical to conclude that other people can also hear them (delusion).

Abnormal perception (deficit theory). An example of theories of abnormal perception assumes that the fundamental problem underlying positive symptoms is the loss of the distinction between relevant and irrelevant stimuli $^{10-12}$. The patient persistently attends to stimuli that should be ignored and generates complex accounts of why these stimuli are important (FIG. 1); thus, the abnormality lies in the patients' attention to the irrelevant stimuli, rather than in their attempt to explain why these stimuli are important. A failure to ignore irrelevant stimuli that result from one's own thoughts and actions might be due to a failure to tag these stimuli as self generated. Probably the most well-developed theory of this kind concerns the idea ${ }^{13}$ that certain positive symptoms are caused by a defect in self monitoring. Strikingly, many of the symptoms associated with schizophrenia involve misattribution of self-generated actions to others (TABLE 1).

Normally one can easily distinguish one's own actions from those of others, even though every act 


\section{Box 2 | Diagnosing schizophrenia}

In the absence of any biological markers, the diagnosis of schizophrenia must be based on clinical interviews that assess signs, symptoms and history. It must be based on a combination of features as many of the individual features can also be found in other disorders. Fifty years ago the diagnosis of schizophrenia varied widely from one location to another. To deal with this problem, standardized diagnostic procedures were introduced, of which the fourth edition of the Diagnostic and Statistical Manual of Mental Disorders (DSM-IV) is currently one of the most widely used. However, these standards are constantly being revised and changed. One of the purposes of diagnosis generally is to provide pointers for aetiology and treatment. In this respect, the value of a diagnosis of schizophrenia remains controversial. The features that follow are necessary for a DSM-IV diagnosis of schizophrenia ${ }^{106}$.

- Characteristic symptoms. Two of the following are needed: delusions, hallucinations, disorganized speech, grossly disorganized or catatonic behaviour, and negative symptoms (that is, affective flattening, alogia and avolition). Note that delusions alone are sufficient if they are bizarre (see TABLE 1) and hallucinations alone are sufficient if they consist of a voice keeping up a running commentary on the person's behaviour/ thoughts or if they consist of two or more voices conversing with each other.

- Social or occupational dysfunction

- Persistent signs of the disturbance for several months

As we write, a number of brave experts are faced with the difficult task of composing the new version of the diagnostic guide - the DSM-V. One of the important problems that current thinking forces them to grapple with is the question of whether we should be thinking in terms of diagnostic categorizations or in terms of the symptoms themselves. Many argue that a diagnosis of schizophrenia is too vague and inexact to be helpful, and that it would be far better to treat our patients and conduct our research according to the presence or absence of specific symptoms. We think that cognitive models such as the one proposed here may be helpful to both the categorical and the dimensional approach, as they offer explanations at the level of the symptom but they also attempt to draw together these explanations to show why symptoms of differing types may occur together and, therefore, why the syndrome exists.

Corollary discharge

The estimate of sensory feedback that is derived from the internal copy of the motor signal (the efference copy).

Efference copy

An internal copy of a motor signal that can be used to

predict the sensory consequences of the movement. one performs has sensory consequences that could just as easily have arisen from an external cause. As Helmoltz ${ }^{14}$ pointed out, an image may slide across the retina because it represents an object that is moving or because the eye is moving while the object is stationary. These two possibilities can usually be distinguished without difficulty, but certain positive symptoms may reflect an inability to make this distinction ${ }^{15}$. Notably, delusions of motor control and auditory hallucinations may respectively reflect tendencies to attribute to external sources active movements and inner speech ${ }^{16,17}$. There is evidence that when a patient hears a voice the source may be their own sub-vocal speech ${ }^{18,19}$ (however, see REFS 20,21).

At the physiological level this ability to discount one's own actions requires a form of self monitoring, dependent on processes such as corollary discharge ${ }^{22}$ and efference copy ${ }^{23,24}$, through which the brain uses the motor commands that generated the action to predict and reduce awareness of the action's sensory consequences. Several experiments have found that patients with positive symptoms fail to attenuate responses to the sensory consequences of their own actions or their own speech ${ }^{25-27}$. As quite a lot is known about the neural basis of corollary discharge $\mathrm{e}^{28}$, this formulation provides a useful link between symptoms and physiology. In addition, this account helps one to understand what it might be like to have certain positive symptoms ${ }^{29}$. If one's experience of the sensory consequences of one's action was not attenuated, then when one made an active movement it would feel like a passive movement. It would feel as if one's action was being driven by an external force.

Abnormal beliefs. Although a failure of self monitoring captures well the features of the passive experiences that characterize many positive symptoms, it does not seem very relevant to delusions; for example, the sudden onset of a belief that my neighbour is trying to poison me does not have an obvious link to abnormal sensation. For these positive symptoms it seems more plausible that the underlying problem lies with the formation of beliefs, rather than with the perceptions from which the beliefs are derived. Although logical reasoning does not seem to be markedly impaired in patients with schizophrenia, there is evidence of problems with probabilistic reasoning. Probabilistic reasoning is associated with a Bayesian approach to the study of belief formation ${ }^{30}$. Within this framework, a belief is the subjective probability that some proposition about the world is true. This probability is continually updated by new evidence. Abnormal belief formation occurs when beliefs are not updated appropriately on the basis of new evidence ${ }^{31}$.

Abnormalities in the integration of new evidence into beliefs have been observed in deluded patients ${ }^{32}$. One well-replicated abnormality is known as 'jumping to conclusions' (REF. 33). In this paradigm, patients are presented with two urns containing red and blue balls. One urn contains $80 \%$ blue balls and the other $80 \%$ red balls. A sequence of balls is taken from one of the urns and the patient must decide which urn has been selected. Deluded patients reach their conclusion on the basis of significantly less evidence than control participants and express more confidence in their decisions $s^{34,35}$. Once a belief has become sufficiently strong, deluded patients show an abnormal bias against disconfirmatory evidence ${ }^{36}$. In addition, patients with delusions abnormally weight potential outcomes when making decisions ${ }^{37}$. These anomalies of probabilistic learning and decision making correspond to clinical observations of delusions. Patients all too easily develop false beliefs, which they then hold with great confidence and immunity to any counter evidence. As described below, these cognitive abnormalities can also be linked to underlying physiology.

Abnormal beliefs in neurological patients: the need for a two-deficit account. Delusional beliefs are not unique to patients with schizophrenia. They can also be found in patients with overt brain damage and are often associated with obvious deficits. For example, patients with paralysis of one arm may believe that the paralyzed arm is not part of them or that they are able to move it. The deficit is clearly not sufficient to create the false belief, as many patients have paralyzed arms without any such belief. Similarly, a minority of patients with memory loss show confabulation, a false belief about what has happened to them in the past. As has been cogently $\operatorname{argued}^{38,39}$, two deficits are necessary to explain these 


\begin{tabular}{|c|c|}
\hline Symptom & Example \\
\hline Audible thoughts & $\begin{array}{l}\text { The patient would think 'I must put the kettle on', and after a pause of not more than one } \\
\text { second would hear a voice say 'I must put the kettle on'. }\end{array}$ \\
\hline Voices arguing & $\begin{array}{l}\text { Patient G.T. heard one voice say 'G.T. is a bloody paradox'; another say 'He is that, he should } \\
\text { be locked up'; and a third say 'He is not, he is a lovely man'. }\end{array}$ \\
\hline $\begin{array}{l}\text { Voices commenting on } \\
\text { one's actions }\end{array}$ & $\begin{array}{l}\text { A voice in a flat monotone describing everything the patient was doing: 'She is peeling } \\
\text { potatoes, got hold of the peeler, she does not want that potato...' }\end{array}$ \\
\hline $\begin{array}{l}\text { An influence on the body } \\
\text { (somatic passivity) }\end{array}$ & $\begin{array}{l}\text { 'X-rays enter the back of my neck, where the skin tingles and feels warm, they pass down the } \\
\text { back in a hot tingling strip about six inches wide to the waist.' }\end{array}$ \\
\hline Thought withdrawal & $\begin{array}{l}\text { 'I am thinking about my mother, and suddenly my thoughts are sucked out of my mind by a } \\
\text { phrenological vacuum extractor, and there is nothing in my mind.' }\end{array}$ \\
\hline Thought insertion & $\begin{array}{l}\text { 'The thoughts of Eamonn Andrews come into my mind. He treats my mind like a screen and } \\
\text { flashes his thoughts on to it like you flash a picture.' }\end{array}$ \\
\hline Thought broadcasting & $\begin{array}{l}\text { 'My thoughts leave my head on a type of mental ticker-tape. Everyone around me has only } \\
\text { to pass the tape through their mind and they know my thoughts.' }\end{array}$ \\
\hline 'Made' feelings & $\begin{array}{l}\text { 'It is not me who is unhappy, but they are projecting unhappiness into my brain. They project } \\
\text { upon me laughter for no reason.' }\end{array}$ \\
\hline 'Made' impulses & $\begin{array}{l}\text { 'It came to me from the X-ray department. It was nothing to do with me, they wanted it so I } \\
\text { picked up the bottle and poured it.' }\end{array}$ \\
\hline $\begin{array}{l}\text { 'Made' volitional acts } \\
\text { (delusions of control) }\end{array}$ & $\begin{array}{l}\text { 'It is my hand and arm that move, and my fingers pick up the pen, but I don't control them. } \\
\text { What they do is nothing to do with me.' }\end{array}$ \\
\hline Delusional perception & $\begin{array}{l}\text { One of the lodgers pushed the salt cellar towards him, and the patient knew that he must } \\
\text { return home 'to greet the Pope who is visiting Ireland to see his family and reward them'. }\end{array}$ \\
\hline
\end{tabular}

The examples are taken from Schneider's first rank symptoms of schizophrenia ${ }^{114}$.

delusions: a primary deficit (paralysis or memory loss) and a failure to suppress the implausible responses that result from this deficit. In the case of neurological patients, false beliefs seem to derive from the coincidence of damage in two locations, with the abnormal belief formation associated with damage to the prefrontal cortex ${ }^{40}$.

A two-deficit account also seems necessary to explain the false beliefs associated with schizophrenia. If a perceptual anomaly was sufficient to generate symptoms such as delusions of control, then the creation of such anomalies in otherwise healthy people should generate false beliefs. Several experiments show that this is not the case ${ }^{41,42}$. However, although a two-factor account may seem necessary to explain these delusions, symptoms reflecting false perception and false beliefs are so intertwined in schizophrenia that a theory relying on coincidental damage seems very unlikely.

\section{The physiological basis of positive symptoms}

The boundaries between perception and belief at the physiological level are not so distinct. An important principle that has emerged is that both perception of the world and learning about the world (and therefore beliefs) are dependent on predictions and the extent to which they are fulfilled. This suggests that a single deficit could explain abnormal perceptions and beliefs. In illustrating this, we begin by considering the physiological basis for hallucinations.

We can ignore self-generated sensations because they are predictable. We have considered the origin of positive symptoms in terms of a failure to distinguish between changes in experience produced by internal actions and those produced by changes in the external world. Important to this distinction is the predictability of one's own actions. A significant difference between selfgenerated actions and something that occurs outside one's control is that in the former case one can predict what will happen. If something is predictable it is easy to ignore and its sensory consequences can be dampened. Thus, the results of one's own movements or speech are ignored because they are unsurprising. The physiological basis for this is conceptually simple: preparation for the motor act entails a prediction of its consequences, and efferent information is sent to the sensory cortex to suppress its response. By contrast, the hallmark of a sensory experience that derives from an external stimulus is that it is not predicted and hence not suppressed. Unpredictable things are difficult to ignore. Indeed, it is crucial to experience them and incorporate them into an updated understanding of the environment.

This simple model makes a clear prediction: the sensory consequences of a self-generated act should elicit less brain activity than the consequences of an externally generated act. This holds true in the motor domain, where individuals have to overcompensate when trying to match a self-produced force to an externally produced one ${ }^{43}$. It also seems to be the case in the auditory domain. One study ${ }^{44}$ showed that the electrophysiological response to hearing one's own speech is smaller than the response to hearing someone else's speech or to hearing one's own speech distorted. This complements data from magnetoencephalography studies, which suggest that self-produced speech 


\section{Box 3 | Do positive symptoms occur in healthy people?}

\section{Strange perceptions and beliefs are present in many people who would not be} diagnosed with mental illness. For example, experiencing auditory hallucinations is relatively common during the transition from waking to sleep or from sleep to waking, and people suffering the stress of bereavement frequently report hearing the voice of, or even seeing, the dead person. Generally these experiences are very brief, and they are often recognized immediately by the subject as illusory.

However, one state in which hallucinatory experiences are prolonged and usually accepted as real is the dream state. Moreover, experiences in the dream state are often bizarre yet accepted without question at the time. Analogies have been drawn between the electrophysiological and neurochemical changes that occur during the transition to rapid eye-movement sleep and the observations of schizophrenia, and attention has also been directed to the striking overlap in experiences associated with the two states ${ }^{107}$. Others ${ }^{108}$ have pointed out that functional-neuroimaging findings suggest that the altered patterns of brain response that occur during dreaming are redolent of those that are observed in schizophrenia, including disruptions in regional connectivity. Perhaps the dream state arises from disruptions in hierarchical Bayesian processing, such that sensory firing is not constrained by top-down prior information and inferences are accepted without question owing to an attenuation of the prediction-error signal from lower to higher levels.

It seems that people spend most of their time with the delusion that they have an accurate representation of the world. Actually, evidence suggests that we are all rather poor at letting our sensory experience update our beliefs, and that we are susceptible to prior beliefs and social constraints that greatly limit our ability to deal with evidence rationally. For most of us, this may be manifest as poor performance when we try to deal with probabilities or as vulnerability to biases as we try to model the world. For the most part, people do not depart from the beliefs of the herd. However, a substantial number of mentally healthy people have beliefs that are recognized by the majority as bizarre. For example, a sizeable number of people believe that they have been abducted by aliens. It has been argued that the experiences that engender this belief may involve hallucinations related to sleep-waking transitions. Another large set of people claim that they can communicate with dead people. These sorts of beliefs (which seem to many to be as bizarre as positive symptoms) are strikingly different from schizophrenia in at least two respects. First, they do not seem to cause the same distress; rather, they perhaps even provide a source of pleasure or pride. Second, they may promote rather than prevent social behaviour, as they are accepted by a sizeable minority of people who provide each other with support. This latter distinction may be an important one: ultimately, the suffering caused by positive symptoms in patients with schizophrenia may manifest in, and arise from, social difficulties. Social conflict and isolation may be important factors that maintain delusions and modify their expression. What distinguishes a clinical case of experiencing positive symptoms from a non-clinical case may be the symptoms' severity, their emotional connotations and the extent to which the environment can support and sustain the individual ${ }^{32,109,110}$.

Another instance in which people without mental illness can develop beliefs that are every bit as bizarre as delusions is termed 'folie a deux'. In this instance, one or more mentally healthy people, usually living in close proximity to the deluded individual, come to share the individual's delusions ${ }^{111}$. Their beliefs typically disappear when the deluded individual is treated or when they are separated from the individual. This phenomenon shows how strongly one's inferences are affected by those of others.

Latent inhibition

The phenomenon whereby a stimulus that has been

previously presented but has not had any predictive value becomes more difficult to associate with an outcome when presented at a later stage at which it does have predictive value. That is, learning related to the pre-exposed stimulus is slow compared with learning related to new stimuli. dampens and delays auditory cortex responses to selfproduced and expected sounds $s^{45-47}$. Notably, the effect is more pronounced with self-produced talking than with self-generated tones ${ }^{48}$.

Unpredictability and disconnectivity as bases for hallucination. If specific positive symptoms, such as auditory hallucinations, arise from the misperception of inner speech, the experience of auditory hallucinations should be associated with activity in areas related to speech, including the auditory cortex. This seems to be the case $\mathrm{e}^{27,49-56}$.
It has been shown that the synchronization of activity in brain areas that are associated with self-produced speech is attenuated in people with schizophrenia, and that this strongly predicts their likelihood of experiencing hallucinations ${ }^{57}$. This relates to another facet of brain activity in schizophrenia: it has been suggested that the core deficit that underlies symptoms such as hallucinations is disturbed connectivity in the brain. Clearly, a prerequisite for sensory damping of self-produced experience is efficient connectivity between motor and sensory regions. Any disruptions in this connectivity might lead to inefficient motor-sensory communication, a failure in the prediction-based attenuation and, ultimately, difficulties in distinguishing internally from externally generated stimuli. Structural observations have provided support for disconnectivity in schizophrenia ${ }^{58}$, and functional MRI has linked abnormal connectivity to hallucinations ${ }^{59}$ (FIG. 2).

\section{Linking perception and belief}

Beyond sensory processing: prediction and learning. Predictability is a useful marker for internally generated actions, but its significance extends to external stimuli, which can also be more or less predictable - we can learn when and where they will arise and with which other stimuli they are likely to be associated. Indeed, as has been pointed out ${ }^{60}$, it would not be useful (and may be harmful) to form a mental association between two stimuli unless the probability of their co-occurrence is greater than would be predicted from the probability of their occurrence in isolation. Thus, for the world not to remain confusing, elemental and disjointed, each perception of a stimulus must update the internal record of its probability (beliefs about the world): when a new stimulus-outcome pairing occurs, prior occurrences of elements of that pair (the stimulus and the outcome) must be evoked if one is to have any meaningful idea of whether it is truly an association or simply a chance co-occurrence. Thus, how one experiences something at a basic sensory level is dependent on one's knowledge of it (expressed in terms of its predictability): each experience is affected by what one believes. Furthermore, the extent to which one updates what one believes is affected by how that experience adds to it. This is, of course, the insight that was captured by Bayes' theorem ${ }^{61}$.

This interaction between experience and belief creates a problem. Experiences that do not challenge a belief system become predictable and are therefore ignored. The advantage of this is that one is spared the need for inefficient and energy-expensive processing of all the sensations with which one is bombarded. The disadvantage is that such a system can become inflexible: strong beliefs lead one to ignore otherwise-informative experiences. An example of this is latent inhibition ${ }^{62}$ : if, through repeated exposure, one increases the probability of a particular stimulus, one can decrease the extent to which it will become associated with another stimulus at a later stage. Thus, the apparently simple process of stimulus perception is itself a learning event and is critical for higher-order associational and inferential processes $^{63}$. The model one builds of the world (beliefs 
about the world) has a big impact on perception. The idea that perception is an inference about the world that combines sensory signals with prior knowledge is an idea that goes back to Helmholtz ${ }^{14}$, but it has recently been provided with a computational basis in the form of Bayesian inference ${ }^{64}$. Thus, an abnormality in perception may arise from an abnormality in one's beliefs about the world, and vice versa.

It is noteworthy that latent inhibition has been shown, under certain circumstances, to be anomalous in schizophrenia. Some studies show that people with

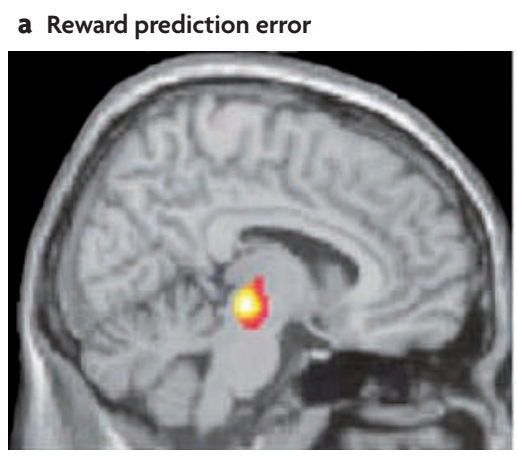

b Causal inference prediction error
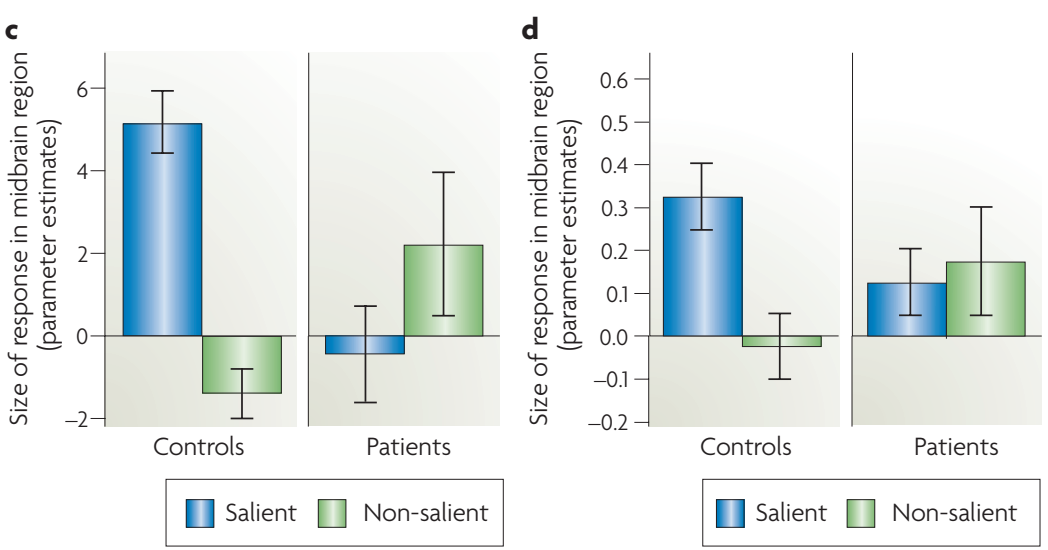

e

"It was as if parts of my brain 'awoke' which had been dormant, and I became interested in a wide assortment of people, events, places, and ideas which normally would make no impression on me. ...The walk of a stranger on the street could be a 'sign' to me which I must interpret. Every face in the windows of a passing streetcar would be engraved on my mind, all of them concentrating on me and trying to pass me some sort of message." Norma MacDonald

Figure 1 | Abnormal response to saliency in midbrain regions of patients with schizophrenia: functional MRI blood-oxygen-level-dependent (BOLD) activity in the midbrain. All data were obtained from a group of 14 people with early psychosis and matched controls. a,b | Functional MRI activity in the midbrain during tasks involving saliency. In one of the tasks ${ }^{75}$ (a), stimuli acted as probabilistic indicators of either financially rewarding ('salient') or neutral ('non-salient') outcomes. In the other ${ }^{76}$ (b), stimuli were part of a causal inferential task and constituted either a violation ('salient') or a fulfilment ('non-salient') of previously learned causal associations. c,d | The size of the effect on the midbrain activation across groups and conditions for each of the experiments described above (c corresponds to $\mathbf{a}$ and $\mathbf{d}$ corresponds to $\mathbf{b}$ ). In both experiments, control subjects' activation was greater for salient than for non-salient events, but this effect was attenuated and/or partially reversed in patients. e $\mid$ An example of what it is like to experience such abnormal saliency ${ }^{112}$. Parts $\mathbf{a}$ and $\mathbf{c}$ are reproduced, with permission, from REF. 75 ( ) (2007) Macmillan Publishers Ltd. All rights reserved. Parts $\mathbf{b}$ and $\mathbf{d}$ are reproduced, with permission, from REF. 76 (C) (2007) Oxford University Press. schizophrenia learn more rapidly than control subjects in response to stimuli to which they have previously been exposed $^{65}$. This rare instance in which the abnormality in schizophrenia is expressed in terms of superior performance also raises the intriguing possibility that this reciprocal interaction between perception and learning may be altered in such patients. It suggests that the distinction between abnormal perceptions and abnormal beliefs is not particularly helpful.

Of course, one does not just make predictions about when stimuli are likely to occur: one also makes predictions about how stimuli relate to each other - for example, as causes and outcomes. The phenomenon of 'blocking' (REF. 66) shows that the tendency to form an association between stimuli or events depends on whether their co-occurrence is surprising. In blocking experiments, a stimulus (A) is associated with a reward (R), and then this stimulus is paired with a new stimulus (X) and the participant continues to receive the reward when the A-X pair is seen. Even though $\mathrm{X}$ has always been seen in association with $\mathrm{R}$, it is not treated as a predictor of reward. Learning the association between $\mathrm{X}$ and $\mathrm{R}$ is said to be blocked: this seems to be because A already fully predicts $\mathrm{R}$ and so nothing new is learned from X.

Blocking demonstrates that what one learns about relationships in one's environment is dependent on one's predictions. Surprise - a mismatch between what is expected and what actually happens - drives learning. In formal models of learning this surprise is referred to as prediction error ${ }^{67}$. It may be extremely important in governing how one updates one's ideas about the world in order to interact with it as safely and as profitably as possible ${ }^{68}$. The partial loss of the blocking effect in schizophrenia (and in people who have taken amphetamines $)^{69}$ suggests that prediction error is abnormal in these patients. In the next section, we consider the physiological basis for this and relate it to findings in schizophrenia.

Prediction error, learning and dopamine. There is strong evidence that dopamine has a role in prediction error-dependent learning in animals, and increasing evidence that this is also the case in humans. Key mesolimbic dopaminergic regions are highly responsive to reward and prediction error ${ }^{70-72}$. Furthermore, reward- and prediction error-related behaviours are sensitive to dopaminergic perturbations. A drug that upregulated dopamine function in healthy participants strengthened error-dependent reward learning, whereas a dopamine-blocking drug reduced such learning ${ }^{73}$. There is also evidence that this system is disrupted in schizophrenia: the ventral striatum (a key component of the mesolimbic dopamine circuit) was shown to be under-active in response to rewarding experiences in patients $^{74}$. Furthermore, abnormal reward-based prediction-error activity has been observed in midbrain and target regions in people with schizophrenia ${ }^{75}$. The patients had a relatively suppressed response to stimuli that should be relevant and important and a relatively augmented response to stimuli that should be neutral 


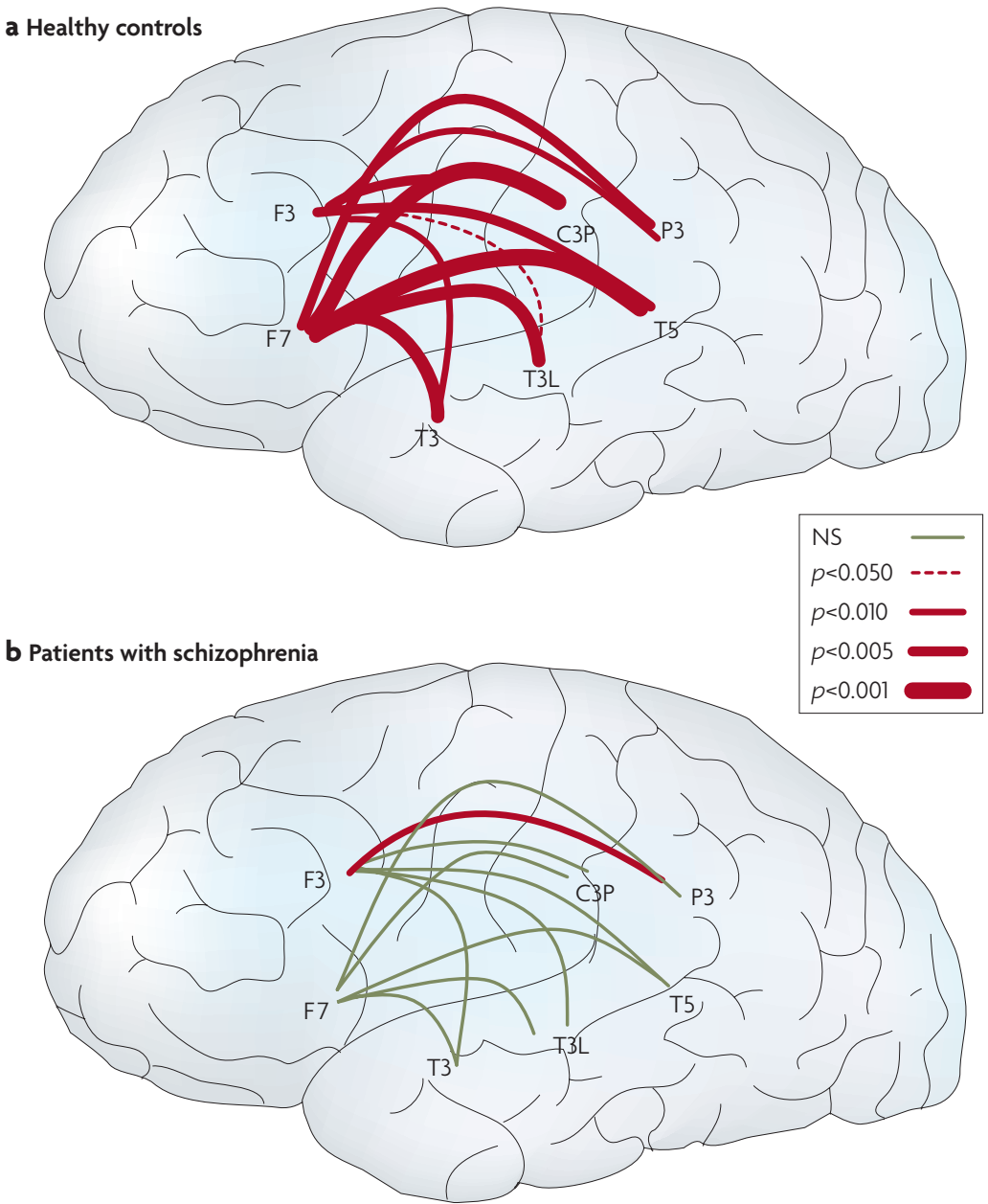

Figure 2 | Abnormal connectivity associated with hallucinations. Lateral views of the left hemisphere of the brain. The red lines connect areas that exhibited greater frontotemporal electroencephalogram coherence during talking than during listening for normal controls and patients with schizophrenia. The thickness of the line indicates the probability level for the $t$-tests that compared the findings. The thicker the line, the larger the difference between the two coherences. In the controls, coherence during talking was greater than during listening for all 20 of the electrode pairs. In the patients, coherence during talking was greater for only two of the pairs (one in each hemisphere). NS, not significant. Figure is reproduced, with permission, from REF. 113 ( ) (2002) Elsevier.

or unimportant. This observation was confirmed in the same patients using a causal inferential task that led to prediction error ${ }^{76}$ and is comparable to that made in another study which used stimuli that predicted aversive rather than rewarding outcomes ${ }^{77}$.

There is thus ample evidence from both behavioural and physiological studies for abnormalities of probabilistic learning in schizophrenia. We suggest that the formation of beliefs about the world can be equated with probabilistic learning. The next step will require us to make a precise link between the learning abnormalities that can be elicited in experimental settings and the nature of the delusions observed by clinicians. For example, increasing dopamine activity increased the rate of learning from gains but had no effect on learning from losses ${ }^{73}$. This asymmetry of learning may explain the pathological gambling that is sometimes associated with treatment with dopamine agonists ${ }^{78}$. An elegant but speculative extension of this idea to schizophrenia proposes that delusions are resistant to being disproved because evidence in favour of the delusion (gains) is counted whereas evidence against the delusion (losses) is discounted as the result of an overactive dopamine system. This is, of course, an exaggerated form of a process that is commonly observed in many people (BOX 3), including neuroscientists developing theories of schizophrenia.

A hierarchy of prediction and model building. It has been proposed that a hierarchical Bayesian system might be a basic principle for brain function ${ }^{79-82}$. According to this theory, building models of the world through prediction errors is a common feature of many brain systems, and these systems are arranged in a hierarchy so that the prediction error emitted by a lower-level system becomes the input for a higher-level system. At the same time, feedback from the higher-level system provides the prior beliefs for the lower-level system. In this framework, the prediction-error signal is a marker that the existing model or inference has not fully accounted for the input. A readjustment at the next level in the hierarchy may increase the accuracy and reduce the prediction error. But if it does not, higher-level readjustments are required. Higher levels provide guidance to lower levels and ensure an internal consistency of the inferred causes of sensory input at multiple levels (FIG. 3).

An example of this interaction between lower- and higher-level systems relates to the allocation of attention. A number of formal associative-learning models ${ }^{83,84}$ acknowledge the importance that prediction error has for allocating attention. Common to these models is the idea that violation of expectation renders an occurrence worthy of greater attention, and that as a result the item becomes more 'salient' and, perhaps, more associable. Therefore, a low-level prediction error may have consequences at a higher level of the hierarchy: the prediction error alerts the organism to the fact that the current model is wrong, suggesting that there has been a change in the environment ${ }^{85}$. If the prediction error cannot be accommodated by the existing model, new learning and new inferences ensue. The possibility that there has been a change may render previous evidence relatively obsolete compared with the new attention-grabbing perceptions.

This framework relates to a series of models concerning how delusions might arise in people with schizophrenia. Miller ${ }^{86}$ discussed the process of delusion formation in terms of an increased willingness to see meaningful associations in the world, whereas Gray, Hemsley and others suggested that the key problem is a diminished ability to integrate new experiences with stored knowledge based on previous experiences ${ }^{87}$. In either case, a consequence might be the development of strange conclusions on the basis of evidence or experience that supported these conclusions only very weakly. As described above, the prediction error strongly signals that there has been a change, leading to a compelling demand for a new inference and a discounting of the 
a

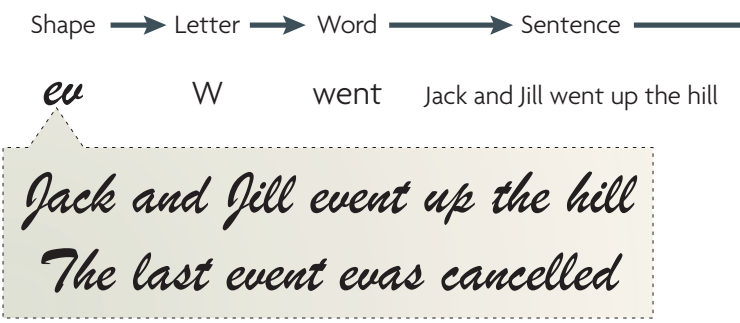

b

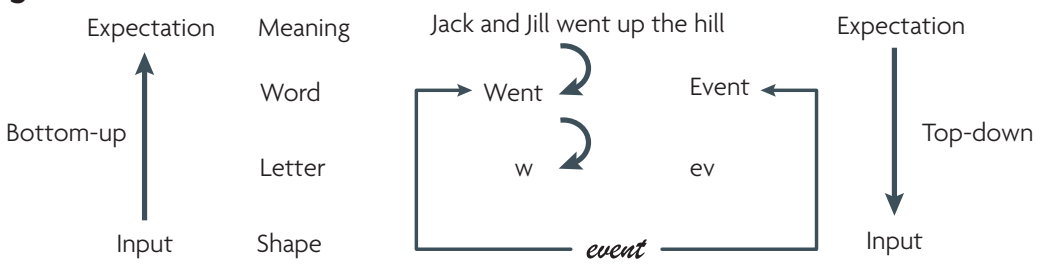

Figure 3 | An illustration of a hierarchical Bayesian scheme for reading. It should be easy to read the sentence 'Jack and Jill went up the hill'. You simply convert the shapes into letters, the letters into words and the words into sentences (a). But in the example shown, the word 'went' looks identical to the word 'event'. So how do you avoid a mistake when you convert these shapes into letters? You use a Bayesian hierarchical processing model (b). Three levels of the hierarchy are shown. At each level, the interpretation of input to lower levels is constrained by expectations at higher levels. In other words, our expectations (also known as priors) determine how we interpret new evidence. Graphic shapes (sensory input) are constrained by likely letters, letter strings are constrained by likely words, and words are constrained by likely sentences. In the example shown, the graphic shape is ambiguous as it could be either ' $w$ ' or 'ev'. Only at the level of the sentence can a prediction error (a deviation from expectation) be generated - the word 'event' does not fit the context of the rest of the Jack and Jill sentence. This is illustrated in $\mathbf{b}$ by the arrow going up from the graphic shape to the word meaning, bypassing the letter stage. The effects of this error are propagated down the hierarchy, such that the shape is constrained to be interpreted as ' $w$ '. This is illustrated in $\mathbf{b}$ by the curled arrows going down from sentence to word and then to shape. Once we know the meaning of the sentence, this changes our perception of the words, letters and shapes. Upwards arrows indicate the bottom-up effects of sensation on interpretation; downwards arrows indicate the effects of expectation on interpretation.

existing model. Relevant to this, Kapur suggested that disrupted dopamine responses account for changes in the salience of a stimulus ${ }^{88}$. In other words, a stimulus that evokes dopamine firing will grab attention and will demand an explanation or an updating of belief, and it is this that forms the germ of a delusional belief ${ }^{12}$. There is behavioural evidence that people with schizophrenia show abnormalities in the assignment of salience to stimuli ${ }^{89,90}$, whereas at the physiological level there is evidence of an augmented brain response to stimuli that, from an experimental point of view, ought to be relatively neutral or non-salient ${ }^{75-77}$. Furthermore, there is evidence that this phenomenon actually relates to the presence of unusual beliefs ${ }^{76}$.

These ideas fit well into the hierarchical Bayesian framework described above. In terms of this framework, the problem that leads to the positive symptoms of schizophrenia starts with false prediction errors being propagated upwards through the hierarchy. These errors require higher levels of the hierarchy to adjust their models of the world. However, as the errors are false, these adjustments can never fully resolve the problem. As a result, prediction errors will be propagated even further up the system to ever-higher levels of abstraction.
The severity of the insult to the Bayesian system may account for how far up the hierarchy a false prediction error will go.

\section{From disrupted neurochemistry to beliefs}

We can now revisit our aim of identifying aberrant physical or neuronal processes and linking these to symptoms. We have suggested that the positive symptoms of schizophrenia may be explained by a disruption in the prediction-error signal. In this section, we consider more closely the possible nature of this disruption, in terms of how it might arise from abnormal dopamine neurotransmission and how this single disruption could be reflected in impairments, comparable in nature but perhaps different in expression, as we move from low-level sensory to higher-level inferential processing.

According to current theories ${ }^{91}$, dopamine-neuron firing encodes the precision or uncertainty of prediction errors and this precision weights the influence of prediction errors on inference. This is crucial for optimizing the balance between top-down prior beliefs and bottom-up sensory evidence. We can therefore speculate on the impact of abnormal dopamine-mediated neuromodulation on prediction errors. It is not the prediction errors per se that are faulty, it is the way that they are used and quantified. The size of the prediction error is meaningless without an estimate of its precision. An analogy is a simple statistical test of a difference. We estimate the size of the difference (which is analogous to the prediction error) together with a measure of its variation (the certainty or precision of the prediction error). The important value is one that takes into account not just the magnitude of the prediction error but also its uncertainty. Thus, a relatively small prediction error might be given undue weight (if the uncertainty is underestimated), leading to a false inference. Alternatively, excessive noise might dilute the effects of even a large prediction-error signal, leading to a reluctance to accept an inference as adequately explaining the input.

At the lower, sensorimotor levels the disturbance could be seen as subtle changes in perceptual and motor function, both of which have been noted in schizophrenia ${ }^{92,93}$. In terms of experience, stimuli might feel unusual, important and salient, leading to a difficulty in allocating attention to appropriate aspects of the environment. This might be experienced as an enhancement of background sounds or sights that should be irrelevant, a phenomenon that is clearly described by patients in the early stages of the illness ${ }^{94}$. As discussed above, a disruption in the prediction error accompanying selfgenerated actions could lead to those actions being felt as strange and externally generated. Furthermore, as well as demanding attention, stimuli accompanied by a large prediction error would become more readily associable, perhaps accounting for the bizarre but compelling coincidences that patients frequently report ${ }^{95}$. Note that this model allows for varying degrees of disruption: a very small alteration in this mechanism, an alteration that we believe to be fundamental to schizophrenia, can push someone over the edge from a state in which 
perception/belief formation is altered but still self correcting to a state in which even increasingly flexible and imaginative inferences no longer accommodate persistent, unreliable prediction error. It is feasible that the deficit may be sufficiently large for delusion formation while being insufficient to produce gross impairments in sensory or motor function.

A noisy prediction-error signal could therefore lead to patients' strange experiences, together with their readiness to accept incidental stimuli and events as important and meaningful and to link them in unusual ways. Persistence of the disruption up the hierarchy can mean that the attempts at the lower levels to explain the world will fail. Achieving a world model that is not continually being signalled as wrong will require more complex changes. The world will feel strange, and there may be a sense that there is some underlying change that must be discovered. This is referred to as a delusional mood. Beyond this, an inference is required to account for the experiences, and now the experience of a strange world may give way to a compelling sense that a particular perception or occurrence has a meaning for the patient (a delusional perception). When this inference is specific and related directly to the self it is referred to as a delusion of reference, a common positive symptom.

Ultimately, someone with schizophrenia will need to develop a set of beliefs that must account for a great deal of strange and sometimes contradictory data. Very commonly they come to believe that they are being persecuted: delusions of persecution are one of the most striking and common of the positive symptoms of schizophrenia, and the cause of a great deal of suffering. If one imagines trying to make some sense of a world that has become strange and inconsistent, pregnant with sinister meaning and messages, the sensible conclusion might well be that one is being deliberately deceived. This belief might also require certain other changes in the patient's view of the world. They may have to abandon a succession of models and even whole classes of models. This dramatic shift was described by Peter Chadwick ${ }^{96}$, a psychologist who wrote compellingly about his experience of a schizophrenic breakdown: "I had to make sense, any sense, out of all these uncanny coincidences. I did it by radically changing my conception of reality." In other words, the false prediction errors (uncanny coincidences) forced him to abandon a high-level model of the world. He ceased to be sceptical about parapsychology and concluded that other people, including radio and television presenters, could see into his mind.

There are several aspects of the richness of the psychotic experience that we believe the model can account for. For example, according to the model, an inner voice is unpredictable and therefore feels alien. Furthermore, persistent abnormal prediction-error signals when the same voice is re-experienced might make a familiar voice seem new: the sufferer might experience this as the presence of multiple voices and perhaps multiple persecutors. The repeated occurrence of the faulty signal in essence may render the world baffling and unreliable, providing ever greater challenges to the sufferer's explanatory models. The resultant inferences become limited in their richness and bizarreness only by the boundaries of the individual's imagination.

\section{Conclusions}

At the beginning of this Review we characterized the difference between hallucinations and delusions as reflecting the difference between perceptions and beliefs. In terms of this distinction the positive symptoms of schizophrenia seem to reflect two underlying abnormalities, suggesting that a two-factor explanation is required. However, recent computational models of perception and learning suggest that the same fundamental mechanism (Bayesian inference), by which a model of the world is updated by prediction errors, applies to both perception and belief formation. There may well be a hierarchy of such inferencing devices in the brain, where lower levels of the hierarchy are more relevant to perception and upper levels are more relevant to beliefs. We suggest that the positive symptoms of schizophrenia are caused by an abnormality in the brains' inferencing mechanisms, such that new evidence (including sensations) is not properly integrated, leading to false prediction errors. The view put forward here has a firm grounding in recent developments in the physiological and computational basis of brain function.

Future research may reveal whether a discrete problem at the lower levels leads to error propagation up a normally functioning hierarchy, or whether the problem lies in a common defect in inference mechanism occurring at all levels of the hierarchy. For example, we might ask whether dopamine is linked to prediction errors at all levels of the hierarchy in the same way. Other, rather daunting challenges remain: for example, how can we explain the relapsing and remitting nature of the disease? We would need, of course, to take into account the impact of anti-dopaminergic medications (for a consideration of this see REF. 12). Another question is why the disorder does not tend to emerge until at least adolescence. One possibility is that the Bayesian inferential processes become more prominent with the maturational processes (including those relating to prefrontal connectivity) that occur during adolescence and early adulthood. Further, although we have deliberately ignored negative symptoms, it is interesting to consider whether this model might have relevance for this extremely incapacitating feature of schizophrenia. We speculate that this deficit could indeed be ultimately responsible for the amotivational, asocial, akinetic state that is characteristic of negative symptoms. After all, a world in which sensory data are noisy and unreliable might lead to a state in which decisions are difficult and actions seem fruitless. We can only speculate on whether the same fundamental deficit could account for both positive and negative features of schizophrenia but, if it could, we suggest that it would be more profound in the case of negative features, and this increased severity might be invoked to account for the strange motor disturbances (collectively known as catatonia) that can be such a striking feature of the negative syndrome. 
1. McGrath, J., Saha, S., Chant, D. \& Welham, J. Schizophrenia: a concise overview of incidence, prevalence, and mortality. Epidemiol. Rev. 30, 67-76 (2008).

2. Crow, T. J. Molecular pathology of schizophrenia: more than one disease process? BMJ 280, 66-68 (1980). An influential paper that made the distinction between positive and negative symptoms.

3. Liddle, P. F. Schizophrenic syndromes, cognitive performance and neurological dysfunction. Psychol. Med. 17, 49-57 (1987). An empirical demonstration that the signs and symptoms of schizophrenia should be described in terms of three dimensions: psychomotor poverty, disorganization and reality distortion (hallucinations and delusions).

4. Jaspers, K. General Psychopathology (Manchester Univ. Press, Manchester, 1962).

The author of this book claimed that positive symptoms were 'not understandable'. We disagree.

5. Frith, C. Editorial: in praise of cognitive neuropsychiatry. Cognit. Neuropsychiatry 13, 1-7 (2008).

6. Kemp, R., Chua, S., McKenna, P. \& David, A Reasoning and delusions. Br. J. Psychiatry 170 , 398-405 (1997).

7. Schneider, K. Clinical Psychopathology (Grune \& Stratton, New York, 1959).

An influential attempt to list symptoms specific to schizophrenia.

8. Wing, J. K., Sartorius, N. \& Üstun, T. B. (eds) Diagnosis and Clinical Measurement in Psychiatry. A Reference Manual for SCAN (Cambridge Univ. Press, Cambridge, UK, 1998).

9. Maher, B. A. Delusional thinking and perceptual disorder. J. Individ. Psychol. 30, 98-113 (1974) The original proposal that delusions are caused by anomalous perceptual experiences.

10. Frith, C. D. Consciousness, information processing and schizophrenia. Br. J. Psychiatry 134, 225-235 (1979).

This paper used the term consciousness when doing so was neither popular nor profitable.

11. Hemsley, D. R. A simple (or simplistic?) cognitive model for schizophrenia. Behav. Res. Ther. 31 633-645 (1993)

12. Kapur, S. Psychosis as a state of aberrant salience: a framework linking biology, phenomenology, and pharmacology in schizophrenia. Am. J. Psychiatry 160, 13-23 (2003).

An influential synthesis of ideas concerning the role of dopamine in the generation of positive symptoms.

13. Feinberg, I. Efference copy and corollary discharge implications for thinking and its disorders. Schizophr. Bull. 4, 636-640 (1978).

The original proposal that positive symptoms, in particular thought insertion, might be caused by a problem with corollary discharge.

14 von Helmholtz, H. Handbuch der Physiologischen Optik (Voss, Leipzig, 1866).

A work of genius that suggested, among many other things, that perception depends on inference.

15. Lindner, A., Thier, P., Kircher, T. T., Haarmeier, T. \& Leube, D. T. Disorders of agency in schizophrenia correlate with an inability to compensate for the sensory consequences of actions. Curr. Biol. 15, 1119-1124 (2005)

16. Blakemore, S. J., Wolpert, D. M. \& Frith, C. D. Abnormalities in the awareness of action. Trends Cogn. Sci. 6, 237-242 (2002)

17. Allen, P., Aleman, A. \& McGuire, P. K. Inner speech models of auditory verbal hallucinations: evidence from behavioural and neuroimaging studies. Int. Rev. Psychiatry 19, 407-415 (2007).

18. Green, P. \& Preston, M. Reinforcement of vocal correlates of auditory hallucinations by auditory feedback: a case study. Br. J. Psychiatry 139, 204-208 (1981)

19. Gould, L. N. Auditory hallucinations and subvocal speech. J. Nerv. Ment. Dis. 109, 418-427 (1949). The first demonstration that hallucinations can be associated with the patient's own speech.

20. Junginger, J. \& Rauscher, F. P. Vocal activity in verbal hallucinations. J. Psychiatr. Res. 21, 101-109 (1987).

21. Green, M. F. \& Kinsbourne, M. Subvocal activity and auditory hallucinations: clues for behavioral treatments? Schizophr. Bull. 16, 617-625 (1990).

22. Sperry, R. W. Neural basis of the spontaneous optokinetic response produced by visual inversion. J. Cogn. Neurosci. 43, 482-489 (1950).
23. von Holst, E. \& Mittelstaedt, H. Das reafferenzprinzip (Wechselwirkungen zwischen Zentralnervensystem und Peripherie). Naturwissenshaften 37, 464-476 (1950)

24. Crapse, T. B. \& Sommer, M. A. Corollary discharge across the animal kingdom. Nature Rev. Neurosci. 9 , 587-600 (2008)

25. Blakemore, S. J., Smith, J., Steel, R., Johnstone, C. E. $\&$ Frith, C. D. The perception of self-produced sensory stimuli in patients with auditory hallucinations and passivity experiences: evidence for a breakdown in self-monitoring. Psychol. Med. 30, 1131-1139 (2000). A demonstration that patients with schizophrenia can tickle themselves.

26. Shergill, S. S., Samson, G., Bays, P. M., Frith, C. D. \& Wolpert, D. M. Evidence for sensory prediction deficits in schizophrenia. Am. J. Psychiatry 162, 2384-2386 (2005)

27. Ford, J. M. \& Mathalon, D. H. Electrophysiological evidence of corollary discharge dysfunction in schizophrenia during talking and thinking. J. Psychiatr. Res. 38, 37-46 (2004).

28. Sommer, M. A. \& Wurtz, R. H. Brain circuits for the internal monitoring of movements. Annu. Rev. Neurosci. 31, 317-338 (2008)

29. Hohwy, J. \& Frith, C. Can neuroscience explain consciousness? J. Conscious. Stud. 11, 180-198 (2004).

An attempt to forge a link between neural processes and subjective experience.

30. Fienberg, S. E. When did Bayesian inference become "Bayesian"? Bayesian Anal. 1, 1-40 (2006).

31. Hemsley, D. R. \& Garety, P. A. The formation and maintenance of delusions: a Bayesian analysis. $\mathrm{Br}$. J. Psychiatry 149, 51-56 (1986). An influential demonstration that patients with delusions have problems with probabilistic inferences.

32. Freeman, D., Garety, P. A., Kuipers, E., Fowler, D. \& Bebbington, P. E. A cognitive model of persecutory delusions. Br. J. Clin. Psychol. 41, 331-347 (2002)

33. Garety, P. A., Hemsley, D. R. \& Wessely, S. Reasoning in deluded schizophrenic and paranoid patients. Biases in performance on a probabilistic inference task. J. Nerv. Ment. Dis. 179, 194-201 (1991).

34. Bentall, R. P., Kaney, S. \& Dewey, M. E. Paranoia and social reasoning: an attribution theory analysis. $\mathrm{Br}$. J. Clin. Psychol. 30, 13-23 (1991).

35. Warman, D. M. Reasoning and delusion proneness confidence in decisions. J. Nerv. Ment. Dis. 196, 9-15 (2008).

36. Woodward, T. S., Moritz, S., Menon, M. \& Klinge, R. Belief inflexibility in schizophrenia. Cognit Neuropsychiatry 13, 267-277 (2008).

37. Heerey, E. A., Bell-Warren, K. R. \& Gold, J. M. Decision-making impairments in the context of intact reward sensitivity in schizophrenia. Biol. Psychiatry 64, 62-69 (2008)

38. Coltheart, M. Cognitive neuropsychiatry and delusional belief. O. J. Exp. Psychol. (Colchester) 60 1041-1062 (2007)

A persuasive demonstration that perceptual anomalies are not sufficient to cause delusions.

39. Coltheart, M., Langdon, R. \& McKay, R. Schizophrenia and monothematic delusions. Schizophr. Bull. 33 642-647 (2007).

40. Turner, M. S., Cipolotti, L., Yousry, T. A. \& Shallice, T. Confabulation: damage to a specific inferior medial prefrontal system. Cortex 44, 637-648 (2008)

41. Cahill, C. Silbersweig D \& Frith C. D. Psychotic experiences induced in deluded patients using distorted auditory feedback. Cogn. Neuropsychiatry 1, 201-211 (1996)

42. Blakemore, S. J., Oakley, D. A. \& Frith, C. D. Delusions of alien control in the normal brain. Neuropsychologia 41, 1058-1067 (2003).

43. Shergill, S. S., Bays, P. M., Frith, C. D. \& Wolpert, D. M. Two eyes for an eye: the neuroscience of force escalation. Science 301, 187-187 (2003).

44. Heinks-Maldonado, T. H., Mathalon, D. H., Gray, M. \& Ford, J. M. Fine-tuning of auditory cortex during speech production. Psychophysiology 42, 180-190 (2005)

45. Curio, G., Neuloh, G., Numminen, J., Jousmaki, V. \& Hari, R. Speaking modifies voice-evoked activity in the human auditory cortex. Hum. Brain Mapp. 9 183-191 (2000).

46. Houde, J. F., Nagarajan, S. S., Sekihara, K. \& Merzenich, M. M. Modulation of the auditory cortex during speech: an MEG study. J. Cogn. Neurosci. 14 1125-1138 (2002).
47. Martikainen, M. H., Kaneko, K. \& Hari, R. Suppressed responses to self-triggered sounds in the human auditory cortex. Cereb. Cortex 15, 299-302 (2005).

48. Ford, J. M., Gray, M., Faustman, W. O., Roach, B. J. \& Mathalon, D. H. Dissecting corollary discharge dysfunction in schizophrenia. Psychophysiology 44 522-529 (2007)

49. McGuire, P. K., Shah, G. M. \& Murray, R. M. Increased blood flow in Broca's area during auditory hallucinations in schizophrenia. Lancet 342, 703-706 (1993). An early demonstration that hallucinations are associated with activity in speech-production regions of the brain.

50. Shergill, S. S., Brammer, M. J., Williams, S. C. R., Murray, R. M. \& McGuire, P. K. Mapping auditory hallucinations in schizophrenia using functional magnetic resonance imaging. Arch. Gen. Psychiatry 57, 1033-1038 (2000)

51. Shergill, S. S., Bullmore, E., Simmons, A., Murray, R. \& McGuire, P. Functional anatomy of auditory verbal imagery in schizophrenic patients with auditory hallucinations. Am. J. Psychiatry 157, 1691-1693 (2000).

52. Ford, J. M. et al. Cortical responsiveness during talking and listening in schizophrenia: an event-related brain potential study. Biol. Psychiatry 50, 540-549 (2001).

53. Shergill, S. S., Brammer, M. J., Williams, S. J., Murray, R. M. \& McGuire, P. K. Mapping auditory hallucinations in schizophrenia using functional magnetic resonance imaging. Arch. Gen. Psychiatry 57, 1033-1038 (2000)

54. Shergill, S. S. et al. Modality specific neural correlates of auditory and somatic hallucinations. J. Neurol. Neurosurg. Psychiatry 71, 688-690 (2001).

55. Shergill, S. S. et al. Temporal course of auditory hallucinations. Br. J. Psychiatry 185, 516-517 (2004).

56. Lennox, B. R., Park, S. B., Medley, I., Morris, P. G. \& Jones, P. B. The functional anatomy of auditory hallucinations in schizophrenia. Psychiatry Res. 100, $13-20$ (2000).

57. Ford, J. M., Roach, B. J., Faustmann, W. O. \& Mathalon, D. H. Synch before you speak: auditory hallucinations in schizophrenia. Am. J. Psychiatry 164, 458-466 (2007)

Evidence that hallucinations are associated with a reduction of long-range connectivity in the brain.

58. Kubicki, M. et al. A review of diffusion tensor imaging studies in schizophrenia. J. Psychiatr. Res. 41, 15-30 (2007).

59. Mechelli, A. et al. Misattribution of speech and impaired connectivity in patients with auditory verbal hallucinations. Hum. Brain Mapp. 28, 1213-1222 (2007).

Structural evidence for connectivity problems in schizophrenia.

60. Barlow, $\mathrm{H}$. The exploitation of regularities in the environment by the brain. Behav. Brain. Sci. 24 602-607; discussion 652-671 (2001).

61. Bayes, T. R. An essay towards solving a problem in the doctrine of chances. Philos. Trans. R. Soc. Lond. 53 370-418 (1763)

A work of genius providing the computational basis for the process of inference that underlies perception and belief formation.

62. Lubow, R. E. Latent inhibition. Psychol. Bull. $\mathbf{7 9 ,}$ 398-407 (1973)

63. Barlow, H. Conditions for versatile learning, Helmholtz's unconscious inference, and the task of perception. Vision Res. 30, 1561-1571 (1990). An important proposal about the mechanism of inference in the visual system.

64. Yuille, A. \& Kersten, D. Vision as Bayesian inference: analysis by synthesis? Trends Cogn. Sci. 10, 301-308 (2006).

Review of the evidence that vision depends on inference.

65. Vaitl, D. et al. Latent inhibition and schizophrenia Pavlovian conditioning of autonomic responses. Schizophr. Res. 55, 147-158 (2002).

66. Kamin, L. J. in Punishment and Aversive Behaviour (eds Campbell, B. A. \& Church, R. M.) 279-296 (Appleton Century Crofts, New York, 1969).

67. Rescorla, R. A. \& Wagner, A. R. in Classical Conditioning II (eds Black, A. H. \& Prokasy, W. F.) 64-99 (Appleton Century Crofts, New York, 1972). The computational basis of learning by association.

68. Schultz, W. \& Dickinson, A. Neuronal coding of prediction errors. Annu. Rev. Neurosci. 23, 473-500 (2000).

An important account of the role of prediction errors in learning. 
69. Jones, S. H., Hemsley, D., Ball, S. \& Serra, A Disruption of the Kamin blocking effect in schizophrenia and in normal subjects following amphetamine. Behav. Brain Res. 88, 103-114 (1997).

70. Hollerman, J. R. \& Schultz, W. Dopamine neurons report an error in the temporal prediction of reward during learning. Nature Neurosci. 1, 304-309 (1998).

71. Mirenowicz, J. \& Schultz, W. Importance of unpredictability for reward responses in primate dopamine neurons. J. Neurophysiol. 72, 1024-1027 (1994).

72. Waelti, P., Dickinson, A. \& Schultz, W. Dopamine responses comply with basic assumptions of formal learning theory. Nature 412, 43-48 (2001).

73. Pessiglione, M., Seymour, B., Flandin, G., Dolan, R. J. $£$ Frith, C. D. Dopamine-dependent prediction errors underpin reward-seeking behaviour in humans. Nature 442, 1042-1045 (2006)

74. Juckel, G. et al. Dysfunction of ventral striatal reward prediction in schizophrenia. Neuroimage 29 409-416 (2006)

75. Murray, G. K. et al. Substantia nigra/ventral tegmental reward prediction error disruption in psychosis. $\mathrm{Mol}$. Psychiatry 13, 267-276 (2007).

Evidence of abnormal neural correlates of prediction errors in schizophrenia.

76. Corlett, P. R. et al. Disrupted prediction error signal in psychosis: evidence for an associative account of delusions. Brain 130, 2387-2400 (2007).

77. Jensen, J et al. The formation of abnormal associations in schizophrenia: neural and behavioral evidence. Neuropsychopharmacology 33, 473-479 (2008).

78. Dodd, M. L. et al. Pathological gambling caused by drugs used to treat Parkinson disease. Arch. Neurol. 62, 1377-1381 (2005)

79. Friston, K., Kilner, J. \& Harrison, L. A free energy principle for the brain. J. Physiol. (Paris) 100, 70-87 (2006).

An important proposal that the brain consists of a hierarchy of Bayesian inferencing devices.

80. Murray, S. O., Kersten, D., Olshausen, B. A., Schrater, P. $\&$ Woods, D. L. Shape perception reduces activity in human primary visual cortex. Proc. Natl Acad. Sci. USA 99, 15164-15169 (2002).

81. Lee, T. S. \& Mumford, D. Hierarchical Bayesian inference in the visual cortex. J. Opt. Soc. Am. A Opt Image Sci. Vis. 20, 1434-1448 (2003).

82. Summerfield, $C$. Koechlin, E. A neural representation of prior information during perceptual inference. Neuron 59, 336-347 (2008).

83. Mackintosh, N. J. A theory of attention: variations in associability of stimuli with reinforcement. Psychol. Rev. 82, 276-298 (1975)

84. Pearce, J. M. \& Hall, G. A model for Pavlovian learning: variations in the effectiveness of conditioned but not of unconditioned stimuli. Psychol. Rev. 87, 532-552 (1980)

85 Courville, A. C. Daw, N. D. \& Touretzky, D. S. Bayesian theories of conditioning in a changing world. Trends Cogn. Sci. 10, 294-300 (2006).
86. Miller, R. Schizophrenic psychology, associative learning and the role of forebrain dopamine. Med. Hypotheses 2, 203-211 (1976)

87. Hemsley, D. R. The development of a cognitive model of schizophrenia: placing it in context. Neurosci. Biobehav. Rev. 29, 977-988 (2005).

88. Berridge, K. C. \& Robinson, T. E. Parsing reward. Trends Neurosci. 26, 507-513 (2003).

89. Murray, G. K. et al. Incentive motivation in firstepisode psychosis: a behavioural study. BMC Psychiatry 8, 34 (2008)

90. Roiser, J. P. et al. Do patients with schizophrenia exhibit aberrant salience? Psychol. Med. 30 Jun 2008 (doi: 10.1017/S0033291708003863).

91. Yu, A. J. \& Dayan, P. Uncertainty, neuromodulation, and attention. Neuron 46, 681-692 (2005).

92. Dakin, S., Carlin, P. \& Hemsley, D. Weak suppression of visual context in chronic schizophrenia. Curr. Biol. 15, R822-R824 (2005)

93. Chen, Y., Levy, D. L., Sheremata, S. \& Holzman, P. S. Compromised late-stage motion processing in schizophrenia. Biol. Psychiatry 55, 834-841 (2004)

94. McGhie, A. \& Chapman, J. Disorders of attention and perception in early schizophrenia. Br. J. Psychiatry 34, 103-116 (1961).

An influential survey of experiences in the early stages of psychosis.

95. Chapman, J. The early symptoms of schizophrenia. $B r$. J. Psychiatry 112, 225-251 (1966)

96. Chadwick, P. K. The step ladder to the impossible: a first hand phenomenological account of a schizoaffective psychotic crisis. J. Ment. Health 2, 239-250 (1993).

A fascinating personal account of a psychotic breakdown.

97. Brown, A. S. Prenatal infection as a risk factor for schizophrenia. Schizophr. Bull. 32, 200-202 (2006).

98. van Os, J., Krabbendam, L., Myin-Germeys, I. \& Delespaul, P. The schizophrenia envirome. Curr. Opin. Psychiatry 18, 141-145 (2005).

99. Burmeister, M., Mclnnis, M. G. \& Zollner, S. Psychiatric genetics: progress amid controversy. Nature Rev. Genet. 9, 527-540 (2008).

100. Steen, R. G., Mull, C. McClure, R., Hamer, R. M. \& Lieberman, J. A. Brain volume in first-episode schizophrenia: systematic review and meta-analysis of magnetic resonance imaging studies. Br. J. Psychiatry 188, 510-518 (2006)

101. Lewis, D. A., Glantz, L. A., Pierri, J. N. \& Sweet, R. A Altered cortical glutamate neurotransmission in schizophrenia: evidence from morphological studies of pyramidal neurons. Ann. NY Acad. Sci. 1003 102-112 (2003).

102. Kapur, S. \& Mamo, D. Half a century of antipsychotics and still a central role for dopamine $D 2$ receptors. Prog. Neuropsychopharmacol. Biol. Psychiatry 27, 1081-1090 (2003)

103. Featherstone, R. E., Kapur, S. \& Fletcher, P. J. The amphetamine-induced sensitized state as a model of schizophrenia. Prog. Neuropsychopharmacol. Biol. Psychiatry 31, 1556-1571 (2007).
104. Jentsch, J. D. \& Roth, R. H. The neuropsychopharmacology of phencyclidine: from NMDA receptor hypofunction to the dopamine hypothesis of schizophrenia. Neuropsychopharmacology 20, 201-225 (1999).

105 Corlett, P. R., Honey, G. D. \& Fletcher, P. C. From prediction error to psychosis: ketamine as a pharmacological model of delusions. J. Psychopharmacol. 21, 238-252 (2007).

106. American Psychiatric Association. Diagnostic and Statistical Manual of Mental Disorders, Fourth Edition (American Psychiatric Association, Washington DC, 1994)

The official definition of schizophrenia.

107. Gottesmann, C. The dreaming sleep stage: a new neurobiological model of schizophrenia? Neuroscience 140, 1105-1115 (2006).

108. Schwartz, S. \& Maquet, P. Sleep imaging and the neuro-psychological assessment of dreams. Trends Cogn. Sci. 6, 23-30 (2002).

109. Birchwood, M. Pathways to emotional dysfunction in first-episode psychosis. Br. J. Psychiatry 182 373-375 (2003)

110. Bentall, R. P., Corcoran, R., Howard, R., Blackwood, N $\Sigma$ Kinderman, P. Persecutory delusions: a review and theoretical integration. Clin. Psychol. Rev. 21 1143-1192 (2001)

111. Shimizu, M., Kubota, Y., Toichi, M. \& Baba, H. Folie a deux and shared psychotic disorder. Curr. Psychiatry Rep. 9, 200-205 (2007).

112. Macdonald, N. Living with schizophrenia. Can. Med. Assoc. J. 82, 218-221 (1960).

113. Ford, J. M., Mathalon, D. H., Whitfield, S., Faustman, W. O. \& Roth, W. T. Reduced communication between frontal and temporal lobes during talking in schizophrenia. Biol. Psychiatry 51, 485-492 (2002).

114. Mellor, C. S. First rank symptoms of schizophrenia. $B r$ J. Psychiatry 117, 15-23 (1970)

\section{Acknowledgements}

C.D.F. is supported by the Wellcome Trust and the Danish National Research Foundation. P.C.F. is supported by the Bernard Wolfe Health Neuroscience Fund and by the Wellcome Trust. We are grateful to K. Friston and E. C. Johnstone for their comments on an earlier draft of this paper.

DATABASES

Entrez Gene: http://www.ncbi.nlm.nih.gov/entrez/query

fcgi?db=gene

COMT INRG1

FURTHER INFORMATION

Paul Fletcher's homepage: http://www.neuroscience.cam. ac.uk/directory/profile.php?pcf22

Chris Frith's homepage: http://www.fil.ion.ucl.ac.uk/Frith/ Interacting Minds: http://www.interacting-minds.net/Sted/ HOME.html

ALL LINKS ARE ACTIVE IN THE ONLINE PDF 